\title{
Angst und Schrecken in Berlin - Fallbeispiel Nr. 5: Melika (22) und Parviz N. (34)
}

Es ist Frühjahr 2016. Familie N. befindet sich derzeit in einem Flüchtlingsheim, in dem ich ebenfalls als Sprach- und Kulturmittler tätig bin. Die Unterkunft liegt gleich am Landesamt. Dort werden unter anderem die Härtefälle untergebracht, von denen bereits im zweiten Fallbeispiel die Rede war. Melika (22), ihr Ehemann Parviz (34) und ihre fünf Kinder sind seit ungefähr acht Monaten in Berlin. Parviz, der in einer Provinz unweit von Mazar-i-Sharif aufgewachsen ist, ging es wirtschaftlich gut, er hatte unterschiedliche Jobs als Maurer und Maler, während sich Melika als Hausfrau um die fünf Kinder kümmerte. Außerdem lebte seine Mutter unweit von ihnen, wie er immer betont. Er erzählt angesichts der Fragehoheit widersprüchliche Flucht- und Lebensgeschichten, um mich in die Irre zu führen. Der anfängliche Enthusiasmus von Melika und Parviz, Interviews zu geben, lässt recht bald nach. Die Audiomitschnitte verwirren sie, weshalb wir uns darauf einigen, dass diese entfallen. Dennoch erlauben sie mir, meine Notizen für die vorliegende Dissertation zu verwenden. Die Rekonstruktion dieses Falles beruht daher auf schriftlichen Aufzeichnungen. Im Rahmen der Interviews verbringen wir über sechs Wochen hinweg knapp zehn Stunden miteinander.

Als sie aus dem Internet erfahren, dass die Außengrenzen Europas offen seien und sogar Busse die Menschen nach Deutschland führen, machen sie sich „einfach auf den Weg“. Beim Erzählen widerspricht sich Parviz immer wieder oder lügt offensichtlich. Melika schaut in diesen Augenblicken verschämt zur Seite. Die Familie verbringt die ersten Monate in Massenunterkünften und muss ständig von einem Camp ins nächste ziehen. Darauf erhalten sie ein ruhigeres und größeres Zimmer, weil Melika nun ihr sechstes Kind erwartet. Immer wenn ich im Heim arbeite und für Familie N. übersetze, wird es turbulent. Ihre fünf kleinen Kinder, zwischen zwei und acht Jahren, spielen laut und unübersehbar im Zimmer der Familie. Melika ist überfordert mit ihnen. Dennoch hat 
sie enormes Glück, dass sie nicht mehr in einer der Massenunterkünfte leben muss, wie andere schwangere Asylbewerberinnen. Sie sind froh, nicht mehr im vorigen Camp zu leben. Dort haben einige männliche Heimbewohner, die der sunnitischen Mehrheitsreligion angehören, an sämtlichen Türen der Unterkunft die Religion der Asylbewerber*innen markiert, um in der Nacht die Angehörigen der Minderheitenreligionen zu überfallen. Es kommt zu Ausschreitungen und Prügeleien. Etliche Personen werden dabei verletzt. Zwar gehören Melika und Parviz der sunnitischen Mehrheitsreligion an, doch Parviz schafft es, dennoch in eine solche Auseinandersetzung verwickelt zu werden. Der Stress und die Widrigkeiten im Leben eines geflüchteten Menschen sind ihnen zwar neu, doch die Behandlung als minderwertige Menschen nicht. Darüber scherzen so manche Afghan*innen, vor allem diejenigen, die eine längere Zeit im Iran verbringen, bevor sie Deutschland erreichen - offensichtlich kommt es auch dort $\mathrm{zu}$ einer Inferiorisierung. Der Humor ist den beiden allerdings ausgegangen, weil sie seit ihrer Ankunft eine Krise nach der anderen bewältigen müssen. Ich werde in dieser chaotischen Zeit häufig zum Vermitteln gerufen. Einmal wird Melika ohnmächtig und Parviz bekommt einen Schreianfall, während die Kinder zuschauen. Da meine Kolleg*innen hoffnungslos überlastet sind, muss ich mich erneut alleine zu ihnen begeben. In letzter Zeit kippt Melika häufiger um. Sie schaut bei jeglichen zwischenmenschlichen Unstimmigkeiten zu ihrem Mann, der sich daraufhin darum kümmert - meist ohne Erfolg. Dann streitet sich das Paar, was ihre Lage nicht erleichtert. Im weiteren Verlauf der Schwangerschaft erkrankt Melika häufiger. Ihr Immunsystem streikt. Oft liegt sie einfach nur da und ist bewegungsunfähig. Zudem ist bei fünf Kindern immer eines krank, das die anderen ansteckt. Deshalb befinden sie sich in einer Unterkunft gleich am medizinischen Zentrum des Landesamts. Sie wird geradezu starr, wenn sie wie ohnmächtig daliegt. In diesen Episoden ist Parviz gezwungen, sich um sie und die Kleinen zu kümmern. Ansonsten betreut sie unermüdlich die Kinder, während er regelmäßig verschwindet und mitten in der Nacht ohne ein Wort zu sagen zurückkehrt. Manchmal, wenn ich sie anspreche, weint sie grundlos. Dann antwortet rasch ihr Mann für sie. Parviz fällt im Flüchtlingsheim immer wieder durch sein unsoziales Verhalten anderen Personen gegenüber und aufgrund der lautstarken Streitereien mit Melika auf. Das Personal möchte natürlich wissen, worum es bei den Streitereien geht. Mein Auftritt gestaltet sich derart, dass ich ins Zimmer komme, Parviz die Hand gebe, Melika zuwinke und mich bei beiden nach ihrem Wohlergehen erkundige. Ich gestalte diese Einstiegsphase so kurz wie möglich, damit die danebenstehenden Kolleg*innen möglichst schnell das Gespräch beginnen können. Bevor der Wortwechsel beginnt, bitte ich beide Parteien darum, nach ein bis zwei Sätzen meine Übersetzung abzuwarten. Mittels dieser Methode 
gelingt die Kommunikation am besten für mich. Eine Sozialarbeiterin stellt dann eine Frage, die ich übersetze. Darauf startet Melika mit einer zusammenhangslosen Aneinanderreihung von Ereignissen und wird zwischendurch von Parviz ausgelacht. Letzterer scheut im Übrigen nicht davor zurück, zwischenzeitlich vulgäre Kommentare zum Besten zu geben. Abgesehen davon, dass sich keiner an meine „Zwei-Sätze-Regel“ hält, muss ich nun für beide Stimmen gleichzeitig übersetzten - soweit der jeweilige situative Rahmen eine sinnvolle Vermittlung überhaupt zulässt. Der Streit zwischen den beiden eskaliert und Parviz verlässt den Raum. Inzwischen haben sich neugierige Heimbewohner*innen vor der Tür versammelt. Sehr oft verfüge ich nicht über die Zeit, mich ausgiebig mit ihnen zu unterhalten, weil im Alltag viel zu erledigen ist, was dazu führt, dass ich bisweilen argwöhnisch angeblickt werde. Nun sehe ich mich verbalen Angriffen Parviz' ausgesetzt. Ich sei ein Rassist und afghanischen Asylbewerber*innen feindselig gesinnt. Die Toleranzschwelle bei Menschen, die selbst permanent unter Beschuss stehen, ist äußerst niedrig. Hinter unbeabsichtigten Fehlschlägen oder Missverständnissen wird daher häufig ein offener oder verdeckter Angriff vermutet. Diese Verhaltensweisen sind vor dem Hintergrund der Verletzungen und Demütigungen des Selbst in der Institution nicht ungewöhnlich, sondern eher absehbar, und dennoch prallen sie nicht ganz ohne Nachklang an mir ab. Die bereits angesprochene emotionale Übertragung (siehe Fallbeispiel 3) vollzieht sich auch in solchen Situationen sehr ausgeprägt. Ein Blick in das Gesicht des wütenden Parviz versetzt mich ebenfalls in Wut. Die Wechselwirkung zwischen Mimik und Emotionen, so etwa der Einfluss der Gesichtsmuskelbewegungen auf das Erleben von Gefühlen (vgl. Facial-Feedback-Hypothese ${ }^{1}$ ), ist für mich deutlich spürbar.

Als Sprach- und Kulturmittler werde ich oft zu Asylbewerber*innen geschickt, die Farsi oder Dari sprechen und melden, dass sie Probleme haben, nicht nur in Unterkünften, sondern auch wahllos auf dem Gelände des Landesamts. Ob es

\footnotetext{
${ }^{1}$ Die Facial-Feedback-Hypothese (für einen Überblick vgl. die Metaanalyse von McIntosh 1996) besagt, dass Emotionen das Ergebnis unserer Mimik sind und korrespondierende Gesichtsausdrücke bzw. mimische Entsprechungen (Emotionsausdrücke) haben. Die Facial-Feedback-Theorie arbeitet in Studien (insbes. Strack et al. 1988) vor allem mit gut gelaunten und wütenden Gesichtsausdrücken, weil dabei jeweils unterschiedliche Muskelpartien des Gesichts zum Einsatz kommen. Der Zygomaticus major und der Risorius-Muskel werden bei guter Laune aktiviert, der Musculus orbicularis oris sowie der Corrugator-Muskel bei schlechter Laune. Die Ergebnisse der Experimente von Fritz Strack zeigen, wie Emotionen durch die bloße Nachahmung eines Gefühls unter Einsatz von Gesichtsmuskeln beeinflusst werden.
} 
sich um ein psychisches, soziales, medizinisches oder sonstiges Problem handelt oder ob eine Qualifizierung meinerseits vorliegt, diese Aufgaben zu lösen, steht gar nicht zur Debatte. Man ist froh, dass jemand vor Ort ist, der die Sprachen beherrscht. Dies trifft vor allem zu Beginn meiner Forschung zu. Alles Übrige geht auf die Verantwortung der jeweiligen haupt- oder ehrenamtlichen Helfer*innen. Im Chaos des Landesamts, wo tagsüber zahllose Asylbewerber*innen umherirren, jeder mit ganz eigenen Anliegen, und Sachbearbeiter*innen von ihren Abteilungsleiter*innen keine schriftlichen Anweisungen erhalten, gehören Regellosigkeit und verwirrende Beliebigkeit zur Agenda der Institution Asyl. Der chronische Mangel an qualifiziertem Personal auf allen Ebenen der Institution Asyl führt dazu, dass so auch Sprachmittler*innen nicht selten in Situationen geraten, die weitaus mehr als sprachliche und kulturelle Kenntnisse erfordern. So werde ich auch zu Melika und Parviz geschickt. Ich erreiche schließlich das Zimmer von Familie N. und höre mir an, was die beiden zu sagen haben. In diesem Fall lasse ich mir Zeit und rede mit ihnen. Sie berichten mir zunächst auf Nachfrage, dass es um Beziehungsangelegenheiten gehe. Doch nach wenigen Minuten benennt Parviz das eigentliche Problem, nämlich ihre Uneinigkeit bezüglich der Auswanderung. Es erscheint mir bizarr, ihnen zu sagen, dass es jetzt zu spät sei, um sich über die Fluchtgründe Gedanken zu machen, und dass sie das Beste aus ihrer gegenwärtigen Situation machen sollten. Daraufhin klingelt Parviz' Handy, mit dem er kontinuierlich während des Gesprächs herumgespielt hat. Seine Mutter ist dran, die via Lautsprecher ein Machtwort spricht; Melika sei die dumme, faule und verwöhnte Frau, die jetzt das bekomme, was sie verdient habe. Für Parviz scheint die Sache nun endgültig geklärt. Melika senkt den Kopf und verstummt. Ihre Kinder spielen für kurze Zeit mit einer Heimmitarbeiterin im Spielraum ein paar Zimmer weiter. Ich vereinbare anschließend einen Termin bei den Psychiater*innen gegenüber im Haus. Sie erhalten stark sedierende Medikamente (wieder einmal Benzos), darüber hinaus wird jedoch nichts unternommen. In den Gesprächen mit Melika und Parviz stelle ich fest, dass er wieder in die Heimat zu seiner Mutter zurückmöchte, weil der Ankunftskontext nicht seinen Erwartungen entspricht. Melika sitzt währenddessen da und äußert lediglich, dass sie in Deutschland bleiben wolle. Ihre Kinder, an die er nicht denke, hätten hier - ihrer Ansicht nach wenigstens eine Zukunft. Die Gespräche enden meist in einer Streiterei und einem entsprechend dramatischen Abgang von Parviz.

Parviz ist dauernd sauer, beleidigt oder aggressiv. Dass gleich mehrere Frauen in seinem unmittelbaren Umfeld Machtpersonen sind, zum Beispiel seine Ärztin, die Heimleiterin, einige Sozialarbeiterinnen etc., passt ihm von Anfang an nicht. Ich begleite ihn beispielsweise einmal zur psychiatrischen Fachkraft. Es dreht sich hauptsächlich darum, dass ihm klargemacht wird, wie schlecht es seiner Frau gehe. 
Er bleibt jedoch uneinsichtig. Im Anschluss an das Fachgespräch schlendert er auf den Korridoren der psychiatrischen Anlaufstelle herum und trifft eine Ärztin, die ihm scheinbar gefällt: Er zeigt auf ihren Ringfinger und macht ein fragendes Gesicht dazu. Die Ärztin ist zunächst verdutzt und nimmt an, es handle sich um ein Missverständnis. Dies geschieht zur selben Zeit, in der ich mit der ärztlichen Fachkraft seine Medikamente herbeischaffe, ein paar Meter vor uns. Nach Parviz' missglücktem Flirtversuch begleite ich ihn zurück zur Unterkunft. Kurz danach vertraut mir Melika an, dass sie sich scheiden lassen will. Sie hat bemerkt, dass Trennungen unkompliziert seien, und möchte Hilfe in Bezug auf ihre Kinder, befürchtet sie doch, diese würden unter den Eltern aufgeteilt. Ich spreche unmittelbar danach mit meinen Kolleg*innen und wir koordinieren ein kurzfristiges Meeting, in dem uns eine anwaltliche Fachkraft versichert, dass Melika im Fall einer Trennung und anschließenden Scheidung in der jetzigen Situation ihre Kinder definitiv behalten könne; wenn Gewalttaten gegenüber der Frau vorliegen, behält die Mutter in der Regel die Kinder. Da Melika schon öfter bedroht und geschlagen wurde, ist diese Bedingung erfüllt. Dass eine anwaltliche Fachkraft im Amt ist, die uns in diesem speziellen Fall hilft, stellt übrigens eine seltene Ausnahme dar. Der Plan ist, Parviz am selben Tag noch in eine andere Flüchtlingsunterkunft zu transferieren und Melika künftig in ein unbekanntes Frauenhaus unterzubringen. Dort wird sie eine Wohnung für sich und ihre Kinder erhalten. Einige Stunden später gehe ich mit meinen Kolleg*innen zu Melika und erkläre ihr die Lage. Sie sitzt auf ihrem Bett. Während einige Sozialarbeiter*innen und ich um sie herumstehen, hält sie sich das Gesicht zu und weint. Zunächst muss ich eine ganze Weile erklären, was überhaupt ein Frauenhaus ist. Aus ihrer Mimik ist erkennbar, dass sie zweifelt. Ich frage nach und sie bejaht: Das Konzept eines Frauenhauses ist ihr völlig schleierhaft. Sie glaubt mir nicht so einfach. Sie sei sich ohnehin nicht mehr sicher, denn eigentlich hänge sie noch an ihrem Partner. Unter einem Vorwand schicken wir ihren Mann, der mittlerweile ins Heim gekommen ist und bemerkt hat, dass etwas Ungewöhnliches im Gange ist, zu einer ärztlichen Fachkraft. Nachdem ich mit viel Erfindungsreichtum und Skizzenzeichnungen auf einem Notizblock elaboriere, was Frauenhäuser sind, und versichere, dass es sie tatsächlich gebe und sie heute noch mit all ihren Kindern in ein solches einziehen könne, willigt sie ein, die Trennung durchzuführen. Sie möchte allerdings noch eine halbe Stunde Zeit für sich. In der Zwischenzeit gehe ich rüber ins Haus M, weil dort eine Sprachmittlung vonnöten ist. Wieder einmal geht es im Landesamt hoch her. Hunderte von Asylbewerber*innen stehen an, um versorgt zu werden. Das übliche Bild wartender Geflüchteter in Schlangen. Von Weitem sieht man ein paar helfende Personen, die Trinkpäckchen und Sandwiches verteilen, meistens aus Spenden unterschiedlicher Supermarkt- und Drogerieketten. Ein Ehepaar schnappt sich Dutzende von 
Trinkpäckchen und marschiert von dannen, während die wartende Gruppe ein großes Palaver veranstaltet und ihren Unmut über die Dreistigkeit anderer äußert. Gerade hat eine betagte syrische Frau einen Herzinfarkt erlitten. Zum Glück sind Krankenschwestern da und der Rettungswagen ist auch schon gerufen. Während wir uns das Geschehen anschauen, stehen meine Kolleg*innen und ich auf den Grünflächen des Landesamts und unterhalten uns über Melikas Situation. Kurz darauf begeben wir uns wieder zu ihr. Sie liegt noch im Bett, hält sich weinend die Stirn fest und erteilt den Kindern willenlose Befehle. Diese ignorieren ihre Mutter und ihre Bitte, still zu sein, völlig. Im Gegenteil verhalten sie sich permanent so, dass sie Aufmerksamkeit erregen. Das ist ungeheuer störend für meine Arbeit, vor allem, weil ich sie ja verstehe und nicht immer und alles ignorieren kann, schließlich sind es Kinder in einer Ausnahmesituation. $\mathrm{Zu}$ meiner Vielfachrolle kommt in solch seltenen Situationen die des Aufpassers hinzu, womit ich jedoch vollkommen überfordert bin. Zwischendurch schluchzt Melika und gibt unverständliche, zusammenhangslose Halbsätze zum Besten - für mich als übersetzende Fachkraft ein zusätzlicher Stressor. Die Kolleg*innen schauen mich in der Erwartung an, meiner Simultanübersetzung von Melikas Lamento relevante Aspekte für die Verlegung ins Frauenhaus entnehmen zu können. Schließlich trifft ihr Gatte ein. Wir bringen ihn ins Zimmer und eröffnen ihm die Entscheidung seiner Frau, woraufhin er vollständig die Beherrschung verliert. Zunächst wird er zornig. Er brüllt, bekommt rote Augen und seine Augenbrauen ziehen sich zu. Er versucht die Kinder auf seine Seite zu nehmen, während er Melika weiter anbrüllt. Tränen laufen ihm die Wangen runter. Mit seinen Händen zeigt er verurteilend auf Melika und schreit heraus, dass er sich umbringen werde, falls sie nicht direkt alles zurücknehme. Danach beginnt er am ganzen Leib zu zittern. Ich übersetze und schaue zu, wie Melika unaufhörlich weint. Eine Sozialarbeiterin umarmt sie. Parviz, der immer noch die zwei ältesten Kinder mit seinen Armen vor ihrer Mutter abschirmt und mit Suizid droht, wird nicht ernst genommen. Ich stehe genau vor ihm und den Kindern, als er plötzlich aus dem offenen Fenster mitten durch die metallenen Rollos springt. Als ich aus dem Fenster schaue, höre ich ihn schreien: „Du Bastard. Du iranischer Bastard. Du hast ihr den Floh ins Ohr gesetzt. Das ist nur deine Schuld, ich werde mich noch rächen, warte nur ab!“ Wir vergewissern uns zunächst, dass es Parviz den Umständen entsprechend gut geht. Er scheint nach dem Sprung vom ersten Stock bewegungsunfähig, aber ansonsten quicklebendig zu sein. Schnell bringe ich die Kinder weg ins Spielzimmer und melde der Heimleiterin, was passiert ist. Das Security-Personal trifft ein, als es zu spät ist. Ein Kollege und ich laufen hinunter zu Parviz. Ein Rettungswagen fährt in diesem Augenblick auf das Gelände des Landesamts, der jedoch nicht für Parviz bestimmt ist. Die Sanitäter*innen laufen auf dem Weg zu Haus M an uns vorbei und fragen 
nach der „syrischen Frau mit Herzanfall“, während sie stutzig auf Parviz schauen. Wir winken ab und zeigen auf das medizinische Zentrum. Wie ich Parviz Flüchen entnehme, denkt er, ich verhindere durch das Abwinken absichtlich seine Bergung durch die Rettungskräfte. Mir erscheint es sinnlos, ihm die Situation zu erklären und deutlich zu machen, dass er sich noch gedulden müsse. Zehn Minuten später erscheint ein zweiter Rettungswagen, der allerdings nicht ganz bis Haus M durchfährt, sondern auf halber Strecke wendet und umkehrt. Wie uns auf einen Anruf kurz darauf berichtet wird, hat der betreffende Sanitäter angenommen, man hätte aus Versehen zwei Rettungswagen wegen des Herzinfarkts zum Haus M gerufen. Etwa fünfzehn Minuten später taucht dann endlich der ersehnte Rettungswagen auf und fährt Parviz ins Krankenhaus. Zwischenzeitlich haben die Heimbewohner*innen, unter ihnen auch die Kinder der Familie N., aus den Fenstern zu uns heruntergeblickt: auf Parviz - nach wie vor regungslos und dennoch ein großes Palaver veranstaltend -, meinen kettenrauchenden Kollegen und mich. Melika geht es so schlecht, dass sie die Unterkunft nicht verlassen kann oder will. Sie nimmt Medikamente und schläft ein. Am nächsten Morgen sagt sie, dass sie es sich anders überlegt habe. Parviz ist frühmorgens vor der Unterkunft aufgetaucht und vor Melikas Fenster ausfallend geworden. Von Verrenkungen und Schrammen abgesehen ist er offenbar kerngesund. Er droht ihr nun ganz offen, aus Rache nach Afghanistan zurückzukehren und ihre Mutter zu töten. Anschließend werde er erneut nach Deutschland kommen, um die eigenen Kinder umzubringen. Dies alles teilt er ihr auch über ihre Tante mit, die dazu eigens aus Brandenburg angereist ist. Wegen eines Hausverbots kann er nicht einfach wieder die Unterkunft betreten. Da der Auszug der Familie N. ohnehin geplant war und eine neue Familie auf das Zimmer wartet, muss Melika ihre Sachen packen und innerhalb weniger Stunden die Unterkunft verlassen. Sie wird gemeinsam mit ihrem Ehemann und den Kindern in eine der berüchtigten Massenunterkünfte Berlins verlegt. Zwei Tage später trifft wiederholt Melikas Tante ein, die mich darum bittet, die Trennung doch durchführen zu lassen. Die Lage von Melika sei furchtbar, Parviz sei unerträglich und sie sei kurz davor, den Verstand zu verlieren. Als ich ihr mitteile, dass ich nichts mehr tun könne und die Melika gebotene Möglichkeit eine Ausnahme gewesen sei, da eine funktionierende Kommunikation und glückliche Umstände innerhalb der Institution dazu geführt hätten, dass augenblicklich Hilfestellung geleistet werden konnte, dies aber nun nicht mehr möglich sei, geht sie erbost und bitter enttäuscht auf und davon. Nach diesem Ereignis erfahre ich nichts mehr von Familie N.

Dieses Fallbeispiel zeigt, auf welche Weise Willkür und Chaos Affekte auslösen, die ihrerseits zur Bildung existenzieller Gefühle der Angst und Verunsicherung beitragen. Entscheidend ist hierbei, dass Melika und Parviz den 
totalitären Merkmalen Willkür und Chaos ausgesetzt sind. Die Ausgesetztheit schließt die forschende Person nicht aus: Ich erlebe Affekte im Feld und in meinem persönlichen Leben zunehmend als sozial relationale und weniger als intrapsychische Zustände, wie man gemeinhin meint. Zudem wird ersichtlich, dass ich als Anderer meines Gegenübers gar nicht anders kann, als zu reagieren. Es stellt sich somit im Grunde gar nicht die Frage, wie ich emotional reagieren soll, sondern es geschieht nolens volens, sobald ich spreche und handle. Mein Feldaufenthalt ist aus diesem Grund von einem unerquicklichen Gefühl überschattet, das ich mit meiner forscherischen Neugier zu kompensieren suche. Mein forscherisches Interesse ist zentral und soll nicht unerwähnt bleiben. Solche Situationen sind hilfreich, um ein Gespür für die Belange und Probleme der Akteur*innen im Feld zu bekommen. In der beschriebenen krisenhaften Lage hätte im Grunde eine psychiatrische bzw. psychologische Fachkraft das Gespräch führen müssen. Andererseits kann ich angesichts des spezifischen Feldes und meiner spezifischen Rolle nicht einfach Hilfe ausschlagen. Durchgehende personale und materielle Knappheit und die aus ihr hervorgehende Mentalität von Willkür und Gesetzlosigkeit, zwei herausragende Merkmale der totalen Institution, resultieren in Situationen, welche die Individuen innerhalb der Institution noch verletzbarer machen. Die anfangs erwähnte Markierung der Religion an den Türen der Unterkunft ist ebenfalls in diesem Kontext zu sehen. So kristallisiert sich eine Struktur unvermeidbarer Asymmetrie in sozialen Beziehungen heraus, die unaufhaltsam und wie ,automatisiert“ Angriffe auf die Insassen der Institution generiert (auch durch andere Insassen). Im Dickicht der Organisationen und Einrichtungen entstehen Situationen, die Sprachmittler*innen oder anderen Bediensteten Vorschub leisten, höchst verletzliche Personen auszubeuten. Das Besondere an dieser Kränkung ist die Tatsache, dass sich Asylbewerber*innen nicht bloß in einem reziproken Abhängigkeitsverhältnis zu anderen Akteur*innen im Ankunftskontext befinden, sondern deren moralischer Willkür ausgesetzt sind. Dieser Missstand und nicht etwa ein Reflexionsdefizit macht es Geflüchteten unmöglich, kritisch auf soziale Ungleichheitsverhältnisse Bezug zu nehmen. Die Frustration afghanischer Geflüchteter angesichts der Willkür von Sprachmittler*innen steht in direktem Zusammenhang mit den soeben erwähnten Merkmalen der Institution. Während meines Feldaufenthaltes am Landesamt wird mir zum Beispiel Anfang 2017 von Arash H. (48), einem afghanischen Asylbewerber, Geld angeboten, um im Gegenzug eine Mietwohnung zu erhalten. Der Familienvater lebt mit seiner Ehefrau und sechs Kindern in einer Massenunterkunft in Tempelhof. Vorher lebten sie in einer Unterkunft in Brandenburg (Eisenhüttenstadt). Mithilfe einer anwaltlichen Fachkraft schaffen sie die Überstellung nach Berlin. Hier finden sie jedoch 
erneut unzumutbare Zustände in Unterkünften vor und werden angesichts ihrer ethnischen Zugehörigkeit (Hazara) von Heimbewohner*innen angegriffen. Auf die Frage, weshalb Arash mir solch ein Angebot mache, antwortet er, eine befreundete Familie habe ebenfalls über eine übersetzende Fachkraft eine Wohnung erhalten können. Arash ist verzweifelt und bereit, sein gesamtes Vermögen für die Vermittlung einer Wohnung einzusetzen. Ich rate ihm, anerkannte Maklerbüros zu kontaktieren und sich nicht auf die Versprechen fremder Dolmetscher*innen zu verlassen. Anderen Dolmetscher*innen passiert dies ebenfalls hin und wieder. Zudem sind Dolmetscher*innen für afghanische Asylbewerber*innen meistens iranischer Herkunft, was nicht selten zu Konflikten und/oder Missverständnissen führt. Besonders mit Blick auf die essentiellen Anhörungen beim BAMF stellt dies eine Herausforderung für alle Beteiligten dar. Die Feindseligkeit zwischen unterschiedlichen ethnischen Gruppen ist hervorstechend und wird, wie in den Fallbeispielen aufgezeigt, durch Dynamiken der Institution angekurbelt (Stichwort ethnische Hierarchisierung). Doch selbst wenn afghanische Sprachmittler*innen übersetzen, werden bisweilen ganze Anhörungen falsch vermittelt, weil persönliche Ansichten die Übersetzung beeinflussen. Solche Anhörungen müssen in der Folge auf Kosten der Steuerzahler*innen wiederholt werden. Oft versetzt dann die Kluft zwischen dem, was die geflüchtete Person gerade aussagt, und dem, was laut Protokoll der ersten Anhörung der Fluchtgrund war, meine Kolleg*innen und mich in Erstaunen. Einmal werde ich sogar für eine Anhörung bestellt, weil die Asylbewerberin aus Afghanistan einen iranischen Dolmetscher verlangt. Afghanische Dolmetscher*innen seien ihr zufolge ,viel rassistischer als die Iraner“.

Die unumgängliche Asymmetrie, welche die Gesamtheit aller sozialen Interaktionen zwischen Gefangenen und Bediensteten der totalen Institution durchzieht, geht Hand in Hand mit einer weiteren Dynamik, die in Anlehnung an den aus dem Versicherungswesen stammenden Begriff „moral hazard“ als moralische Versuchung bzw. moralisches Risiko im Asylkontext bezeichnet werden kann. Durch die konsequente Vermeidung langfristiger Pläne zur Versorgung von Geflüchteten in der Bundesrepublik entstehen systematisch Aushöhlungen in der Institution Asyl, die, wenn überhaupt, durch unqualifizierte Kräfte in unangemessenen Kontexten ausgefüllt werden. Diese Lücken beziehen sich insbesondere auf reale Arbeitsplätze und Positionen innerhalb der Institution Asyl, so etwa im BAMF. Sie sind jedoch auch symbolisch zu verstehen, quasi als blinde Flecken eines Systems, das ,automatisiert" seine Gefangenen verletzt. Auf der Mikroebene des Alltags manifestiert sich dies beispielsweise in Defiziten bei menschenrechtlichen Standards, fairen Asylanhörungen, einer angemessenen medizinischen und psychiatrischen Versorgung, Kindergarten- und Schulplätzen, 
Sommer- und Winterkleidung usw. Afghanische Asylbewerber*innen werten solche Ausgrenzungsmechanismen in der Regel als absichtlich herbeigeführte Angriffe auf ihre Person. Totalitäre Strukturen lösen systematisch Angriffe (und Gegenangriffe) aus, die unkontrollierbar sind, ist einmal die Maschinerie der totalen Institution in Gang gesetzt worden. Daraus ergibt sich eine Dynamik, die opportunistisches Verhalten seitens der Bediensteten sowie Misstrauen und Feindseligkeit auf Seiten der Geflüchteten evoziert. Konkret besteht das moralische Risiko, wenn man das Konzept des „moral hazard“ auf den Asylprozess anwendet, darin, dass angesichts kurzfristiger Strategien in der Flüchtlingspolitik Individuen innerhalb der Institution keine Anreize haben, vorgegebene Richtlinien, sofern überhaupt vorhanden, zu befolgen. Dies gilt bemerkenswerterweise sowohl für Bedienstete als auch für Gefangene. Qualifiziertes Personal wird in sämtlichen Bereichen des Asylprozesses immer seltener. Bedienstete der Institution Asyl erhalten temporäre Arbeitsverträge und arbeiten unter prekären Verhältnissen. Viele Sachbearbeiter*innen im Landesamt werden für einige Monate vom Jobcenter ausgeliehen und müssen anschließend zurück. Auch das BAMF sucht so Lücken zu füllen. Um demnach innerhalb der totalen Institution die jeweiligen Ziele erreichen zu können, etablieren sich überwiegend destruktive Handlungsweisen als Anpassungsformen. Schädliche Handlungsformen vermischen sich mit Interessenkonflikten und Informationsasymmetrien ${ }^{2}$ innerhalb des vom moralischen Risiko beherrschten Asylprozesses. Ein weiterer wichtiger Punkt, der sich am Merkmal der Willkür und den aus ihm resultierenden Phänomenen festhalten lässt, ist die weitreichende und existenzielle Art des Einflusses auf die betroffenen Individuen. Die totale Institution Asyl setzt nicht bloß eine Disziplin des Handelns voraus, sondern vielmehr eine Disziplin des Seins. Totalitäre Merkmale und daraus entstehende schädliche Praktiken - also die erwähnten „Anpassungen“ der Gefangenen auf beiden Seiten - zielen darauf ab, durch unaufhörliche explizite und implizite Angriffe auf das Selbst des Menschen dessen Essenz „neu zu schreiben“. Das Selbst des Gefangenen soll nicht nur modifiziert oder bearbeitet, sondern unter willkürlich stattfindenden Angriffen wesentlich umgemünzt werden. Goffman (1973: 184 ff.) beschreibt die „Disziplin des Seins" in der Verpflichtung, einen bestimmten Charakter zu haben und unterscheidet in der Folge primäre und sekundäre Anpassungsformen:

\footnotetext{
${ }^{2}$ Darunter die fehlenden schriftlichen Anweisungen und die sich ständig ändernden Regeln in den Behörden.
} 
„Nun kann ein Begriff neu eingeführt werden. Wenn ein Mensch auf kooperative Art und Weise eine verlangte Tätigkeit für eine Organisation leistet - in unserer Gesellschaft unterstützt durch die institutionalisierten Standards der Wohlfahrt, den durch Belohnungen und gemeinsame Werte geschaffenen Antrieb und durch die Androhung vorgesehener Strafen -, dann verwandelt er sich in einen Mitarbeiter; er wird zum ,normalen', ,programmierten' oder zugehörigen Mitglied. In angemessenem Geist gibt und bekommt er das, was für ihn vorgesehen ist, gleich ob dies viel oder wenig von ihm selbst verlangt. Kurz, er stellt fest, dass von ihm offiziell verlangt wird, nicht mehr und nicht weniger als das zu sein, worauf er vorbereitet wurde, und in einer Welt zu leben, die ihm tatsächlich kongenial ist. Unter diesen Bedingungen möchte ich davon sprechen, dass das Individuum eine primäre Anpassung an die Organisation unternimmt [...]" (Goffman 1973: 185).

Unter sekundärer Anpassung versteht Goffman im Unterschied dazu ein Verhalten, mit dem sich die Insassen von ihrer zugewiesenen Rolle distanzieren können. Die sekundäre Anpassung stellt eine Möglichkeit dar, wie das Individuum sich der Rolle und dem Selbst entziehen kann, welche die Institution für es verbindlich hält“" (ebd.). Denn Handlungen im vorgeschriebenen Geist auszuführen, bedeutet gleichsam zu akzeptieren, dass man eine bestimmte Art von Mensch ist, der in einer bestimmten Art von Welt lebt (Steinert 1973: 101). Mit sekundären Anpassungen können sich Gefangene von vorgeschriebenen Identitäten, Rollen, Selbsten und den mit ihnen entstehenden Emotionen lösen. Goffmans Anpassungsbegriff wurzelt zudem darin, dass Institutionen durch gezielte und übermäßige Angriffe auf das Selbst nicht bloß eine Disziplin der Handlungen, sondern eine Disziplin des Selbst erfordern. Willkürlich ausgeführte Handlungen seitens des Personals mit dem Ziel, die Gefangenen zu disziplinieren (,streng sein mit denen"), münden in Infantilisierungsmechanismen. Bedienstete fühlen sich für die Insassen verantwortlich, womit eine Art funktionalistische Moral in der Institution aufkeimt, die es den gefangenen Menschen aufzuerlegen gilt:

„Kurz, jede institutionelle Perspektive enthält personalisierte Moralvorstellungen, und in jeder totalen Institution entwickelt sich im Kleinen etwas, das einer funktionalistischen Moralauffassung sehr ähnlich ist. Die Übersetzung des Insassenverhaltens in moralistische Termini, die mit der anerkannten Perspektive der Anstalt übereinstimmen, enthält notwendig pauschale Verallgemeinerungen hinsichtlich der Natur des Menschen. Angesichts der Tatsache, dass das Personal für die Insassen verantwortlich ist und ihnen eine bestimmte Behandlung angedeihen lassen soll, neigt es dazu sich eine Theorie der menschlichen Natur zurechtzulegen. Als impliziter Teil der institutionellen Perspektive rationalisiert diese Theorie das Handeln, sie hilft den sozialen Abstand von den Insassen einzuhalten, unterstützt stereotype Vorstellungen über letztere und rechtfertigt die ihnen zuteilwerdende Behandlung“(Goffman 1973: 90). 
Der Begriff „Disziplin“ erscheint allerdings zu weich, denn Intensität, Dauer und Bedingungen der Angriffe auf das Selbst konvertieren den unterworfenen Menschen in ein ganz und gar beschädigtes Individuum. Ein angeschlagenes Individuum kann weniger Energie für den Widerstand gegen Ungerechtigkeiten im Machtgefüge des Ankunftskontextes aufwenden. Unter Widerstand fällt etwa die Teilnahme an Demonstrationen und anderen gesellschaftspolitisch relevanten Aktivitäten, die auf menschenrechtswidrige Dynamiken in der Asylpolitik hinweisen. Der menschenrechtliche Kern des Asylgesetzes und der gesamten Institution Asyl wird somit ausgehöhlt und sinnentfremdet. Dieser Gesichtspunkt ist im Rahmen der Reflexion über Emotionen im Asylprozess nicht zu vernachlässigen: Die Ummünzung des Selbst in der Weise, wie sie innerhalb der Institution Asyl geschieht - insbesondere in Anbetracht der Tatsache, wie subtil und heimtückisch unterschiedliche Angriffe auf das Selbst anmuten -, steht dem humanitären Wesen dessen, was Asyl im Prinzip bedeutet, diametral entgegen. Dieses Paradoxon zwischen dem, was die Institution faktisch tut, und dem, was offiziell als ihr Leitsatz gilt, bildet das Fundament für sämtliche Interaktionen des Personals und der Gefangenen innerhalb der Institution. Goffman beschreibt dieses Paradoxon ebenfalls, bezieht es jedoch ausschließlich auf ,die tägliche Aktivität des Personals“ (1973: 78). Tatsächlich bedeutet die grundlegende Widersprüchlichkeit in der totalen Institution Asyl jedoch weitaus mehr, weil sie auf beiden Seiten eine Atmosphäre von Doppelmoral und Dehumanisierung schafft, die in zunehmendem Maße zur Normalität wird. Individuen, die ihr Menschenrecht auf Asyl - AEMR Art. 14 - wahrnehmen möchten, werden ironischerweise mit diesem Paradoxon, das anderen modernen Demokratien auch nicht fremd ist, überrascht.

Ein weiterer Gesichtspunkt bei der aggressiven Konvertierung des Selbst innerhalb der beschriebenen Konstellation bezieht sich auf die Rolle der Asylbewerber*innen innerhalb der im Ankunftsstaat herrschenden sozialen Ordnung. Die soziale Distanz oder „kastenähnliche Aufteilung“ (McEwen 1980: 157) zwischen „Flüchtlingen“ und „Deutschen“ definiert die emotionale Grundstimmung der Asylbewerber*innen. Die gleich zu Beginn erfahrene Atmosphäre von Willkür und Chaos formt die Auffassung vieler meiner Kontakte, dass „ohnehin alle Deutschen so seien“. Die Gespräche während der vielen Wartezeiten drehen sich oft darum, wie man solch totalitäre Dynamiken, die ausdrücklich als beabsichtigt empfunden werden, begegnen kann. Hilflosigkeit und Wut kommen auf und determinieren eine gewisse Mentalität im Ankunftskontext, welche die künftig zu spielende unterlegene Rolle der Fluchtmigrierenden („Knechte“) im sozialen Gefüge Deutschlands antizipiert. Das Konzept von „Integration“ und die Diskussion darüber werden entsprechend ironisch verstanden. Ganze Stunden 
verbringen meine Kontaktpersonen und ich in den verschiedensten Wartesituationen scherzend damit, wie absurd etwa die Vorstellungen über Integration in vielen öffentlichen Medien anmuten. In einem Gespräch mit Cihan (21), einem afghanischen Asylbewerber, kommt dies deutlich zum Ausdruck. Während wir in einer Warteschlange stehen, berichtet er von seinen Erfahrungen. Das Gespräch findet Ende 2016 statt.

\begin{abstract}
„Immer dieses Gerede von Integration. Integration hier, Integration dort (...). Immer dasselbe. Und wenn es darauf ankommt, grenzen die uns aus. Die wollen einfach nicht, dass wir Teil der deutschen Gesellschaft werden, das ist doch offensichtlich. Ich will doch arbeiten, ich will mich doch an das Gesetz halten (...). Aber ich soll mich den ganzen Tag in einem Zimmer mit drei Syrern aufhalten und darf ansonsten nichts. Und wenn ich mich beschwere, sagen die $^{3}$ sowas wie ,ist doch besser als Krieg ‘. Ansonsten muss ich dauernd von einem Termin zum nächsten. Das ist alles. Das ist mein Leben hier.“
\end{abstract}

Nur selten begegne ich einer afghanischen Person im Feld, die tatsächlich daran glaubt, in Deutschland integriert zu werden. Sogar anerkannte Geflüchtete belächeln durchweg das Konzept der Integration. Die Herstellung sozialer Distanz zu Geflüchteten und damit die Konstituierung der gewünschten sozialen Ordnung stellt eine Praxis ihrer Inferiorisierung dar, im Sinne einer „Naturalisierung“4 der sozialen Verhältnisse innerhalb des Ankunftskontextes. Für eine moderne Demokratie ist diese Spaltung in „Kasten“ allerdings als Ausformung einer sozialen Pathologie zu verstehen.

Parviz wiederholt häufig, dass er gar nicht weiß, weshalb er in Deutschland ist. Er erzählt, wie alle, die es sich einigermaßen leisten konnten, plötzlich nach Europa aufbrachen. Die Grenzen waren ja offen. Melika hat sich ihrer Auskunft nach ebenfalls einfach der Fluchtbewegung angeschlossen. Parviz hatte ursprünglich gar nicht die Absicht, auszuwandern, ließ sich aber dennoch überreden.

\footnotetext{
${ }^{3}$ Cihan meint an dieser Stelle die Mitarbeiter*innen der Institution Asyl.

${ }^{4}$ Ich verwende diesen Begriff in Anlehnung an Karl Marx, der in seiner Analyse des Fetischcharakters der Ware aus dem Kapital zu zeigen versucht, dass in einem kapitalistischen System das gesellschaftliche Produkt der Arbeit, der Tauschwert der Ware, als „natürliches“ Merkmal der Dinge erscheint. Marx begründet mithilfe des Naturalisierungseffektes sozialer Verhältnisse, dass soziale Akteur*innen Ungleichheiten und Ungerechtigkeiten als ,gegeben“ hinnehmen und nicht bezweifeln. Die Naturalisierung sozialer Verhältnisse spielt auch bei den Überlegungen anderer kritischer Gesellschaftstheoretiker*innen eine entscheidende Rolle (z. B. Horkheimer, Adorno, Habermas und Honneth).
} 
Darüber klagt er täglich. Aus diesem Gefühl heraus streitet er sich mit Melika, die seiner Meinung nach eindeutig an der Misere der Familie Schuld ist. Der Ankunftskontext ist das Gegenteil von jenem Schlaraffenland, das sie sich vorstellten, als sie herkamen. Die Narrative, es gebe für jede in die europäischen Staaten fliehende Familie bei der Ankunft ein Haus und ein Auto gratis, treffen nicht zu und erweisen sich als eine Fallgrube in der jeweiligen Fluchtgeschichte. Dies alles trägt zum Ehrverlust Parviz' bei. Hier beginnt er zu verstehen, dass er seine ehemalige Rolle aufgeben muss, um zu überleben. Die Peinlichkeit, von seiner Frau verlassen zu werden, und dies vor Personen, die er nicht respektiert, erlebt Parviz als zerschmetternd. Das daraus resultierende Gefühl der Ausweglosigkeit, die Angst, einsam zu bleiben, sowie die erlebte Demütigung stellen die Grenze des Tolerierbaren für ihn dar. Dies treibt ihn in die Verzweiflung. Hier auf deutschem Boden besitzt er keine Kontrolle mehr. Die Macht, über seine Frau(en) zu verfügen, ist ein zentraler Aspekt dessen, was für Parviz, der in einer Provinz unweit von Mazar-i-Sharif aufgewachsen ist, als „männlich“ gilt. Männer müssen sich im Kontext Afghanistans nicht vor anderen Frauen rechtfertigen, besonders nicht mit Blick auf existenzielle Angelegenheiten wie die Ehe. Hinzu kommt die grundsätzliche Skepsis am westlichen Lebensstil. Sein Unverständnis darüber, was sie überhaupt in Deutschland zu suchen haben, und sein Wunsch, wieder in den Heimatkontext zurückzukehren, ist als eine Facette des Gefühls der Überforderung mit dem Ankunftskontext zu deuten. Parviz' Verhalten ist so zu verstehen, dass der Ankunftskontext eine deutliche Modifikation der Emotionsregulierung mit sich bringt, und zwar bei beiden Personen. Die Normen und Werte des Heimatkontextes werden in Deutschland stark infrage gestellt - so sehr, dass Parviz' Identität bedroht zu sein scheint. Die stückweise Aufreibung seiner Persönlichkeit über viele Wochen hinweg empfindet er als Provokation. Er kreiert angesichts der Resonanz seiner sozialen Interaktionen (Katz II) eine Narration (Katz III), die seinem Dilemma im Ankunftskontext einen Sinn verleiht. Mit der Narration eines eigensinnigen und respektlosen Familienvaters wirkt er seinen Ängsten entgegen und lässt seinen Druck in Beleidigungen und Abwertungen seiner Frau sowie anderen Personen gegenüber ab. Die aufkommende existenzielle Unsicherheit formiert einen bestimmten Selbst- und Weltbezug, der wiederum den Möglichkeitsraum seiner Handlungen einengt (vgl. Abschn. 4.2.4). Unsicherheit, Zweifel, Misstrauen, Frust und Verachtung fügen sich zu existenziellen Gefühlen zusammen und kommen in Situierungen innerhalb eines gewissen Spektrums von Möglichkeitsräumen zum Ausdruck. Parviz' existenzielle Gefühle befinden sich nicht „in“ seiner Psyche; sie werden auch nicht ,an ihn herangetragen“, sondern manifestieren sich als Vollzugsform seiner Existenz per se. Seine Gefühle sind seine Seinsweise. Sein dissoziales Verhalten und die entsprechenden 
Widerfahrnisse sind untrennbar mit den Affekten innerhalb der Institution verschränkt. Mithilfe der Narration erreicht er zunächst sein Ziel, indem er seine Frau erpresst und damit die Trennung verhindert. Parviz setzt seine Leiblichkeit kunstvoll und theatralisch ein, um seine Gefühle auszudrücken. Sein Körper, den er aus dem Fenster wirft, stellt sich als Prisma seiner sozialen Realität dar. Sein abwertendes Verhalten Melika gegenüber kennzeichnet seine emotionalen Ausdrucksweisen. Die Befürchtung, vor anderen gleichgesinnten Afghan*innen in Berlin, vor allem jedoch vor den Verwandten aus der Heimat das Gesicht zu verlieren, ist erdrückend und trägt entscheidend zu seiner Verzweiflung bei. Die massive Normverletzung Melikas, die neu gewonnene Freiheit für eine Trennung bzw. Scheidung nutzen zu wollen, ist durch bestimmte Narrative im Ankunftskontext in höchstem Maße negativ belegt. Das ist nicht nur bei Melika so (vgl. Fallstudie Nr. 3). Im weiteren Verlauf meiner Forschung begegne ich dieser Beziehungskonstellation und den entsprechenden dazugehörigen Narrationen regelmäßig: Afghanische Asylbewerber*innen stigmatisieren Frauen, die sich von den traditionellen Werten in Sachen Ehe distanzieren möchten, indem sie sie als Prostituierte bezeichnen. Diese Abwertung geht einher mit der Aufwertung eigener kultureller und religiöser Ideale aus vergangenen Zeiten. Der plötzliche Umbruch in Parviz' Gefühlslage im Augenblick, bevor er springt, stellt eine bemerkenswerte Episode meiner Feldforschung dar, nicht bloß aufgrund der Theatralik der Inszenierung seiner Verzweiflung, sondern weil das Ereignis eine wesentliche Reflexion in Gang gesetzt hat, die mich das Feld, meine Rolle darin und vor allem meine gesamte Forschung fortan aus einem neuen Blickwinkel sehen lässt. Mir wird insbesondere die schwierig in Worte zu fassende existenzielle und „unheimliche“ Komponente des Dritten Raumes bewusst. Hier reflektiert sich die „Unheimlichkeit" in den durch kulturelle Differenzen erzeugten Spannungen und Ambivalenzen und wirkt auf seltsame Weise noch Jahre später nach. Die aufeinandertreffenden Kulturen sind in sich und im Verhältnis zu dem jeweilig „Anderen“ different. Kultur und Identität gehen in einem unaufhörlichen Prozess der dynamischen Auseinandersetzung mit dem jeweils „Anderen“ hervor, wobei der unberechenbare und nicht vertrauenswürdige „Flüchtling“ für die kulturelle Autorität des Aufnahmelandes ein „unlösbares Problem“ (vgl. Bhabha 2000: 51) kultureller Differenz schafft.

Durch die Differenz zum kulturell differenten „Anderen“ spaltet sich im Dritten Raum die Identität Parviz'. Plötzlich wirbeln neue Ideen, Sichtweisen und Lebensauffassungen in dessen Lebenswelt und bilden unüberwindbare Differenzen. Seine Identität ist von totalitären Dynamiken der Institution, aber auch von dem Wunsch seiner Frau, sich vom Patriarchat zu emanzipieren, bedroht. Damit stellt Parviz eine der wenigen denkwürdigen Ausnahmen 
meiner Forschung dar, zumal er ständig betont, dass er in seine Heimat zurückkehren möchte. Die Identitätsbedrohung durch den Emanzipationswunsch seiner Partnerin wurzelt darin, dass der Dritte Raum das Potenzial hat, zum Medium sozialer Interaktionen (Bachmann-Medick 1998: 23) zu werden, aus dem heraus die „Subalterne“ eine Stimme für sich findet. In der Verunsicherung aller Beteiligten eröffnen sich ungeahnte Wege. Bhabha betont ganz im Sinne der doppelten Wirkungsweise von Differenz, dass hybride Grenzerfahrungen Affekte auf beiden Seiten aufkeimen lassen (Bhabha 2000: 303). Die Panik betrifft hier ebenfalls „beide Seiten“, einerseits Melika und Parviz - jede Person auf eine eigene Art - und andererseits die dominante Kultur. Auf gewisse Weise werden im Raum „da-zwischen“ somit die Identitäten aller in Zweifel gezogen. Diese Tatsache gewinnt auf der psychopolitischen Ebene insofern an Bedeutung, als der Dominanzkultur identitätsverändernde Herausforderungen bevorstehen. Die dominante Gesellschaft steht vor Umbrüchen kultureller Differenzen, die ihre Konstrukte von Nation, Heimat und Identität radikal herausfordern. Diese Herausforderung scheint dabei so stark zu sein, dass eher die Gefahr durch als der tatsächliche Umgang mit „den Anderen“ im Vordergrund steht. Die existenzielle politische Frage zielt insofern auf die Analyse des Gefahrenpotenzials „,der Anderen“ ab, ganz im Sinne von „Is your other 'capable' of killing me?" (Höller/Bhabha 2005: 274). Die innere Zerrissenheit Parviz' führt dazu, dass dieser sich zunächst selbst spaltet und dabei symbolisch tötet. Angst und Schrecken resultieren hieraus, weil „der Andere“ im Zeitalter der Globalisierung einerseits vollkommen normalisiert ist, besonders in den Metropolen, und andererseits doch „so radikal unverständlich“ (Bhabha 2012: 56) bleibt. Minderheiten und ihre kulturellen Vorstellungen sind nämlich in der Dominanzkultur eingebettet und nicht mehr ins Externe zu verschieben (Struve 2013: 179). Der Aspekt des „Eingebettet-Seins“ veranschaulicht zudem ein wesentliches Moment der Hybridität, weil sich in ihm unter anderem die komplexe Beziehung von Anerkennung und Ablehnung zwischen „Minderheitenkultur“ und „,dominanter Kultur“ herauskristallisiert (ebd.). Konzepte wie Toleranz und Multikulturalismus $^{5}$ sind laut Bhabha (Höller/Bhabha 2005: 276) irreführend für das Verständnis solcher Konstellationen, weil sich in ihnen weiterhin festgefahrene universalistische (Multikulturalismus) oder partikularistische Sichtweisen (Toleranz)

\footnotetext{
${ }^{5}$ Der Begriff Multikulturalismus verweist auf eine neu rezipierte Formation gelockerter Bindungen von Nationalstaaten an Nationalkulturen, die sich in einer Parallelität unterschiedlicher einzelner Kulturen innerhalb derselben Nationalstaaten ausdrückt (Reckwitz 2001: 179).
} 
manifestieren, die jedoch keine wirklich differenzierte und anerkennende Artikulation jener kulturellen Differenz erlauben. Letztlich verstummt Melika und nimmt die Chance zur Trennung doch nicht wahr, obwohl sie den Mut aufgebracht hat, den Emanzipationsschritt zu wagen. Darin besteht in der vorliegenden Deutung ihre eigentliche Unterwerfung.

Die aus dem Zwischenraum betrachtete Geschichte von Melika und Parviz zeigt, dass alte polarisierende „Containerkonzepte“ von Kultur und Identität nicht in das postkoloniale Denken passen. Im Dritten Raum Berlins werden durch das unentwegte Neuinterpretieren und Neuaushandeln der eigenen kulturellen Identität Differenzen produziert, und zwar sowohl inter- als auch intrakulturell. Infolgedessen wird der gefangene Mensch unter Rückgriff auf Lacan dauerhaft, in einer sich niemals abschließenden Bewegung des Identifikationsprozesses, entfremdet (vgl. Abschn. 4.5). Der gefangene Mensch ist genötigt, sich im Dritten Raum neu zu erfinden. ${ }^{6}$ Melika und Parviz werden als janusköpfige Figur des Dritten Raums interpretiert. Der innere Zwiespalt dieser janusköpfigen Figur wird aus diesem Blickwinkel durch die verschiedenen Charaktereigenschaften, Wünsche und Emotionen beider Personen verkörpert. Melika beabsichtigt, sich der dominierenden Kultur anzupassen, um Emanzipation in Form von Freiheit zu erlangen und ihre eigene Kultur laut Parviz zu ,,hintergehen“. Dieser Konflikt wird durch ihr soziales Drama veranschaulicht. Kultur ist weder statisch noch natürlich, sie ist stetig im Werden. Das Prinzip der „Unreinheit“ von Kulturen, also deren grundlegender Mischcharakter, wie ihn Bhabhas Kultur- und Identitätsbegriff formuliert, wird in dem inneren Konflikt dieser Figur auf besondere Art exemplifiziert, denn sie hat in vielfacher Weise zwei Gesichter. Es gibt nicht die afghanische Kultur, nicht einmal die afghanische Kultur in der Diaspora. Neben dem Mischcharakter zweier unterschiedlicher Subjekte versinnbildlicht der Januskopf dieser Fallstudie verschiedene Facetten der Unheimlichkeit des Dritten Raums als „Kampfplatz“ der Übersetzung kultureller und politischer Natur (Struve 2013: 131).

In diesem Kapitel wurde die emotionale Erfahrung des Asyls von Melika und Parviz geschildert. Ihre Geschichte ist in einem Rahmen beschrieben worden, der aufzeigt, wie Individuen in der Institution Asyl unterschiedlichen

${ }^{6}$ Ideologien und Konzepte des homogenitätsorientierten Multikulturalismus, wie sie durch den Anerkennungstheoretiker Charles Taylor oder den kanadischen Politikwissenschaftler Will Kymlicka vertreten werden, verhindern gerade solche Prozesse der Verwirklichung des Potenzials eingewanderter Menschen. Der Multikulturalismus fixiert Kulturen, Identitäten und selbst Differenzen und postuliert damit, Identität sei immer durch den kulturellen Hintergrund determiniert. Vgl. auch die Multikulturalismuskritik von Kenan Malik (2008). 
unhintergehbaren Dynamiken und Dimensionen unterworfen sind. $\mathrm{Zu}$ diesen zentralen und unverwechselbaren Dimensionen gehören die Rolle der Asylbewerber*innen im sozialen Gefüge Deutschlands, die auf die Ummünzung des Selbst abzielende Disziplinierung des Seins sowie das Paradoxon, das aus der Kluft zwischen Asylpraxis und humanitärem Kern des Menschenrechts auf Asyl entsteht. Diese Dimensionen konstituieren die Architektur des gesamten Asylprozesses mit und prägen eine totalitäre, inhumane Atmosphäre aus. Infolgedessen sind Asylbewerber*innen nicht in der Lage, sich adäquat zu ihren Emotionen in Beziehung zu setzen, zumal bei etlichen abgelehnten Personen die Stigmatisierung als psychisch krank hinzukommt. Vor dem Hintergrund der genannten Dynamiken ist auch die Episode im Büro der Sachbearbeiterin zu verstehen (Intermezzo). Mit der Hervorhebung dieser Szene soll nicht der Eindruck erweckt werden, das Landesamt sei voller chauvinistischer und bösartiger Personen. Ich habe ebenfalls Bedienstete kennengelernt, die sich deutlich und glaubwürdig von fremdenfeindlichen Gesinnungen zu distanzieren wissen. Die beliebige Art und Weise jedoch, wie ein Abteilungsleiter gewünschte Vorgehensweisen im Landesamt kommuniziert, um selbst definierte Ziele zu erreichen, oder wie Bedienstete, die unter Druck stehen, eigenständig Strategien entwickeln, um finanzielle, personale und bürokratische Lücken auszugleichen, ist geradezu paradigmatisch für die Abläufe und Prozesse innerhalb der Institution. Mit Rücksicht auf die Diskrepanz zwischen Personal und Gefangenen, die unumgängliche Asymmetrie in den sozialen Beziehungen innerhalb der Institution und die alldurchdringende Tendenz der Bediensteten, angesichts des ,Staatsversagens“ Asylbewerber*innen selbstständig zu „erziehen“ (,streng sein mit denen") und zu infantilisieren, zeichnet sich eine regimeartige Herrschaftsform in der Institution Asyl ab, die auftretende Emotionen und emotionale Ausdrucksweisen massiv beeinflusst. Automatisierte und systematische Angriffe auf das Selbst konstituieren innerhalb dieses affektiven Regimes eine spezielle Couleur typischer Affekte, die Versuche der Emanzipation unhintergehbar mit einem Prozess der Unterwerfung verknüpfen. Selbst wenn Anpassungsstrategien (primäre und sekundäre Anpassungen gemäß Goffman) angewendet werden, um totalitäre Dynamiken abzumildern, ist man der Totalität des Asylprozesses ausgesetzt. Die grundlegende Unterwerfung, von der in diesem Kapitel die Rede ist, muss von alltäglichen Demütigungen differenziert werden. Demütigungen im Asylprozess bilden lediglich einen Teilaspekt des Unterwerfungsprozesses in der Institution. Neben der fehlenden Privatsphäre in den Unterkünften (Camps) sind die ständige Überwachung durch das Personal, die strukturelle Missachtung des geflüchteten Menschen als Subjekt und die daraus resultierende Aushöhlung des menschenrechtlichen Kerns von Asylgesetzen, das moralische Risiko 
im Asylsystem, das Ausgesetztsein, die alles durchdringende Fragehoheit und die Inferiorisierung totalitäre Merkmale in dieser Fallstudie. Dabei stellt die Inferiorisierung des gefangenen Menschen geradezu die Bedingung seiner Existenz dar, das heißt, sie ist integraler Bestandteil des gegenwärtigen Asylsystems. Ihre Funktion besteht unter anderem darin, die Sichtbarkeit und damit auch Anerkennbarkeit verletzter Individuen im Keim zu ersticken. Durch Dynamiken wie das moralische Risiko wandelt sich zudem die Art der Angriffe bzw. Verletzungen so, dass diese zufälliger und unberechenbarer werden. Dadurch ergibt sich eine zusätzliche Dimension der Totalität. Das affektive Regime Asyl greift aus diesem Blickwinkel zu „affektiven Waffen“, die ihre Gefangenen durch automatisierte Angriffs- und Unterdrückungsmechanismen verletzen und kleinhalten. 


\subsection{Intermezzo: „Happa happa machen“}

Im Sommer 2016. Ich befinde mich in einer vergleichsweise kleinen Notunterkunft für Geflüchtete in Berlin. Im Flur steht ein Glaskasten und gleich daneben ist das Büro der Bediensteten. Es ist schon am frühen Abend, und mein Kollege, der für das Abendessen verantwortlich ist, befindet sich noch in einem Telefonat mit seiner neuen Partnerin. Ich sitze im Glaskasten und helfe Amin Y. (23), einen Antrag - genau gesagt zwei Anträge in einem - auszufüllen. Selbst die Antragsformulare der Institution stecken voller List und Tücke. Die Übersetzung der Begrifflichkeiten und Konzepte, die der deutschen Bürokratie entsprungen sind, bringen mich zum Schmunzeln. Meine Interviewpartner*innen kennen gewöhnlich ihren Geburtstag nicht. Das ist auch bei Amin der Fall. In vielen Ländern des Nahen und Mittleren Ostens wird Geburtstagen keine Beachtung geschenkt. Dies führt dazu, dass die deutsche Bürokratie ihnen den 01.01. als offiziellen Geburtstag zuweist. Nun haben in Berlin wahrscheinlich so viele Menschen am 1. Januar Geburtstag wie in keiner anderen Stadt auf der Welt. Jedenfalls ist der Bezirk Berlin-Mitte für alle, die im Januar geboren sind, zuständig. Amins Berichten zufolge gleicht er

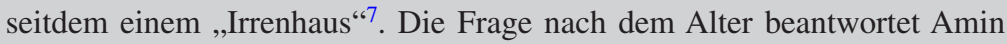
etwa mit „20 halt“ ${ }^{\star 8}$. In seinem Ankunftsnachweis steht, er sei 24 Jahre alt, in der vorläufigen Aufenthaltsbescheinigung wird er als 23-Jähriger ausgewiesen. Ein Haufen weiterer Anträge der Heimbewohner*innen liegt auf dem Personaltisch und wartet darauf, bearbeitet zu werden. Amin ist verzweifelt, weil sein Bruder in eines der unbeliebten Balkanländer abgeschoben werden soll. Darüber hinaus steht Ali (57) klopfend am Glaskasten. Er benötigt sein Codein, um ruhig zu bleiben. Während ich aus dem Nebenzimmer die Codein-Tabletten bringe, die in einem unverschlossenen Schrank liegen, höre ich plötzlich ungewöhnliche, metallene Geräusche im Flur. Schnell drücke ich eine Tablette aus dem 20er Pack heraus, reiche sie Ali in die Hand und verlasse den Glaskasten, um nachzuschauen was überhaupt los ist. Der Kollege, der für das Austeilen des Abendessens

\footnotetext{
ديو انه خانه7

بيست ديخه8
} 
verantwortlich ist, läuft den Korridor entlang und klopft deutlich hörbar für alle mit einem Löffel auf einen Messingtopf. Langsam öffnen sich schon die Türen, und man sieht, wie die Heimbewohner*innen aus ihren Zimmern rausschauen, um in Erfahrung zu bringen, was die Ursache des Tumults ist. Besagter Kollege läuft weiter und schreit aus voller Kehle heraus: „Happa happa! Kommt happa happa machen!“ Ich stehe unweit dieses Spektakels vor der Glaswand. Erstaunt von dem, was vor sich geht, beobachte ich die verschiedenen Gesichtsausdrücke der Menschen, die mehr oder minder gleichzeitig aus ihren kleinen Zimmern heraustreten und halb besorgt und halb erschrocken auf den Kollegen schauen, der diese Handlung offenbar als humorvolle Geste versteht. „Kommt happa happa machen, dann ist erst mal alles jut, ne?" Dann schaut er mich von Weitem an und grinst. Dabei geht er auffallend langsam, beinah feierlich und blickt wie bei einer Prozession nach links und rechts zu den Bewohner*innen. Seine Züge haben etwas Gutmütiges und Naives. Anschließend teilt er das Abendbrot aus.

Open Access Dieses Kapitel wird unter der Creative Commons Namensnennung 4.0 International Lizenz (http://creativecommons.org/licenses/by/4.0/deed.de) veröffentlicht, welche die Nutzung, Vervielfältigung, Bearbeitung, Verbreitung und Wiedergabe in jeglichem Medium und Format erlaubt, sofern Sie den/die ursprünglichen Autor(en) und die Quelle ordnungsgemäß nennen, einen Link zur Creative Commons Lizenz beifügen und angeben, ob Änderungen vorgenommen wurden.

Die in diesem Kapitel enthaltenen Bilder und sonstiges Drittmaterial unterliegen ebenfalls der genannten Creative Commons Lizenz, sofern sich aus der Abbildungslegende nichts anderes ergibt. Sofern das betreffende Material nicht unter der genannten Creative Commons Lizenz steht und die betreffende Handlung nicht nach gesetzlichen Vorschriften erlaubt ist, ist für die oben aufgeführten Weiterverwendungen des Materials die Einwilligung des jeweiligen Rechteinhabers einzuholen.

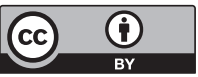

